\title{
Unusual bilateral retrobulbar abscess in the rabbit
}

\section{Norbert Czubaj, ${ }^{1,2}$, Jakub Kliszcz ${ }^{3}$, Jacek Sobczyński ${ }^{4}$, Aneta Gralak ${ }^{5}$}

1 Department of Morphological Sciences, Faculty of Veterinary Medicine, Warsaw University of Life Sciences, Warsaw, Poland Funded by KNOW (Leading National Research Centre) Scientific Consortium "Healthy Animal - Safe Food", decision of Ministry of Science and Higher Education No. 05-1/KNOW2/2015, Warsaw, Poland

Veterinary Clinic "Pulsvet, Warsaw, Poland Veterinary Clinic "Bemowo", Warsaw, Poland

Faculty of Veterinary Medicine, Warsaw University of Life Sciences, Student, Warsaw, Poland

\section{OBJECTIVES}

Retrobulbar abscesses are common problem in rabbits. Typically they have odontogenic origin. One of the most characteristic features of this disease entity is unilateral exophthalmos. Differential diagnosis includes: retrobulbar neoplasia, cellulitis, prolapse of retrobulbar fat, salivary mucocele, thymoma or other mass within thoracic cavity.

We present a case of exophthalmos in a pet rabbit suspected as bilateral abscess of the lacrimal gland.

\section{METHODS}

Female pet rabbit was presented for dental consultation with bilateral asymmetrical exophthalmos and eye discharge. Previous treatment involved eyedrops (diclofenac, tobramycin), analgesia (tramadol) and antibiotics (penicylin and streptomycin). Dental examination and X-rays in various views were unremarkable (Fig. 1). Dual phase CT examination was performed (CereTom ${ }^{\circledR}$, 8-slice portable scanner)

\section{RESULTS}

CT examination showed bilateral abscess in the retrobulbar region with thinning of the alveolar bulla, more profoundly on the left side (Fig. 2). Due to deterioration of the clinical signs the surgery was performed via intraoral approach. Last 4 left maxillary cheek teeth, including alveolar bulla were extracted. No connection between abscess capsule and the bulla was noticed, but lots of pus and detritus was drained. The socket was left for healing by secondary intention. Due to persisting purulent discharge from the wound decision was made for subsequent surgeries - bilateral resection of the zygomatic arch with abscess capsule dissection (Fig. 3).

\section{STATEMENT (CONCLUSIONS)}

Although no histopathological examination was performed most likely diagnosis was lacrimal gland abscess. $\mathrm{CT}$ is a great tool in describing pathologies of a dental and retrobulbar disease in rabbits.

\section{Free-floating fat in a furry! A mobile necrotic lipoma in the abdomen of a guinea pig}

\section{Chris Webb}

Locum Veterinary Surgeon, High Wycombe, United Kingdom

\section{OBJECTIVES}

To report a mobile intra-abdominal necrotic lipoma in a male guinea pig.

\section{METHODS}

An adult male neutered guinea pig (age approximately 3 years) was presented for routine health check after being adopted. Recent history was unremarkable though physical examination revealed an incidental non-painful, firm, highly mobile, bi-convex mass in the mid abdomen.

Repeat examination 2 months later revealed no palpable changes to the mass, though investigation was sought as he had become less interactive. Ultrasound examination revealed an approximately $60 \mathrm{~mm} \times 40 \mathrm{~mm}$ mass with heterogenous architecture and a thin hyperechoic rim.

A detached, ovoid, brown, irregular mass with a thin friable exterior was identified and removed via a midline laparotomy without the need for ligation.

\section{RESULTS}

Histopathological examination revealed the mass was almost entirely composed of necrotic adipose tissue. Adipocytes were well differentiated and the necrotic outlines of the cells were retained. Multifocal areas of necrotic fibrous connective tissue and necrotic debris admixed with degenerate neutrophils were noted. Multi-focally, small to moderate amounts of mineralised material was present. The mass was surrounded by a thin rim of fibrous connective tissue and diagnosed as a necrotic lipoma.

\section{STATEMENT (CONCLUSIONS)}

Several reports of intra-abdominal mobile encapsulated adipose tissue in cows have been described with comparisons being made to those found in the subcutis of extremities in humans.

To the authors knowledge this is the first case of a free-floating necrotic lipoma in the coelomic cavity of a guinea pig. 